\title{
Aspirin-triggered resolvin D1 inhibits TGF-ß31-induced EMT through the inhibition of the mTOR pathway by reducing the expression of PKM2 and is closely linked to oxidative stress
}

\author{
YU LIU* ${ }^{*}$ XIAOLONG YUAN, WENHUI LI, QIANQIAN CAO and YUSHENG SHU* \\ Department of Cardiothoracic Surgery, Clinical Medicine College of Yangzhou University, \\ Subei People's Hospital, Yangzhou, Jiangsu 225001, P.R. China
}

Received July 1, 2015; Accepted August 17, 2016

DOI: $10.3892 /$ ijmm.2016.2721

\begin{abstract}
Transforming growth factor- $\beta 1$ (TGF- $\beta 1$ ) is a potent stimulator of the epithelial-to-mesenchymal transition (EMT), which is a key event in the initiation of tumor cell metastasis. Aspirin-triggered resolvin D1 (AT-RvD1) is known to be involved in the resolution of inflammation; however, whether AT-RvD1 exerts effects on TGF- $\beta 1$-induced EMT remains unclear. Thus, we first explored the effects of AT-RvD1 on the EMT of lung cancer cells. Treatment with TGF- $\beta 1$ increased the level of reactive oxygen species (ROS) and reduced the expression of nuclear factor (erythroid-derived 2)-like 2 (Nrf2). The expression of E-cadherin in A549 lung cancer cells was reduced, and the expression of vimentin was enhanced. AT-RvD1 enhanced the expression of E-cadherin in a concentration-dependent manner and suppressed the expression of Nrf2 and vimentin. AT-RvD1 did not affect the proliferation of A549 lung cancer cells whereas it suppressed the TGF- $\beta 1$-induced migration and invasion of A549 cells. The expression of pyruvate kinase M2 (Pkm2), which is associated with TGF- $\beta$-induced factor homeobox 2 (TGIF2), was significantly increased during the TGF- $\beta 1$-induced EMT of A549 lung cancer cells. The mTOR pathway is known to regulate the expression of various glycolytic enzymes including Pkm2. We examined the involvement of the mTOR pathway in the suppressive effects of AT-RvD1 on Pkm2 expression in A549 cells. The mTOR activator restored the expression of $\mathrm{Pkm} 2$ and partially downregulated the expression of E-cadherin. However, the mTOR activator had no clear effect on the TGF- $\beta 1$-induced EMT. These results suggest that AT-RvD1 is closely linked to oxidative stress and partially
\end{abstract}

Correspondence to: Professor Yusheng Shu, Department of Cardiothoracic Surgery, Clinical Medicine College of Yangzhou University,Subei People's Hospital,98 Nantong West Road, Yangzhou, Jiangsu 225001, P.R. China

E-mail: 475625278@qq.com

*Contributed equally

Key words: TGF- $\beta 1$-induced epithelial-to-mesenchymal transition, aspirin-triggered resolvin D1, lung cancer, reactive oxygen species, pyruvate kinase M2 inhibits TGF- $\beta 1$-induced EMT through the inhibition of the mTOR pathway by reducing the expression of $\mathrm{Pkm} 2$.

\section{Introduction}

Lung cancer accounts for over one million deaths each year worldwide (1). Tumor recurrence and metastasis are the most common events occurring after resection and lead to mortality (2). The causes of tumor recurrence and metastasis have not yet been completely elucidated. With increasingly severe environmental pollution, high concentrations of aerial irritants, such as asbestos or fine particulate matter, in the outdoor air have resulted in an increase in the prevalence of malignant pleural mesothelioma and lung cancer (3). This has focused our attention on the association between inflammation and cancer.

Inflammation is a definite cancer-causing factor, as has been revealed by clinical and epidemiological studies (3). Indeed, an estimated 20-25\% of all types of cancer worldwide are associated with inflammation (4). Neutrophils are regarded as playing principal roles in inflammation-associated tumor development and progression; reactive oxygen species (ROS) and nitric oxide (NO), which are derived from cells involved in the inflammatory response such as neutrophils, are key molecules that stimulate tumorigenesis. ROS are known to initiate and promote cancer (5-7).

The epithelial-to-mesenchymal transition (EMT) occurs during embryogenesis, tissue repair, fibrosis and tumor metastasis (8). The early events of metastasis begin with the EMT, which transforms epithelial cells into elongated, motile and invasive phenotypes (9). The analysis of a large number of lung tumor specimens has shown that the majority of primary lung cancer and even premalignant lesions are characterized by the mesenchymal phenotype (10). Transforming growth factor- $\beta 1$ (TGF- $\beta 1$ ) is a cytokine with multiple functions and is widely accepted to be a profibrogenic factor (11). TGF- $\beta 1$ also plays various roles in the development of diseases, including cancer $(12,13)$. The TGF- $\beta 1$-induced EMT enables cells to acquire migratory and invasive properties. ROS have been considered as contributors to the TGF- $\beta 1$-mediated pathology (14). In view of this, we suggest that ROS may also be associated with tumor metastasis. 
Nuclear factor (erythroid-derived 2)-like 2 (Nrf2) plays a crucial role in the regulation of antioxidant gene expression. One of its most important functions is to encode for ROS scavenging enzymes, such as superoxide dismutase (SOD) and glutathione (GSH) peroxidase (15).

A recent study (16) has shown that Kelch-like ECH-associated protein 1 (Keap1)-Nrf2-GSH signaling, which is regarded as an effective modulator of TGF- $\beta 1$-induced EMT changes, suppresses Nrf2 activity and increases the level of ROS following treatment with TGF- $\beta 1$ in normal cells. In addition, it has been demonstrated that the nuclear translocation level of pyruvate kinase M2 (Pkm2), an alternatively spliced variant of the pyruvate kinase gene that results in the cancer-specific Warburg effect, is enhanced by EMT, leading to the acquisition of invasive behavior (17). ROS have also been demonstrated to inhibit the expression of $\mathrm{Pkm} 2$ through the oxidation of $\mathrm{Cys}^{358}$, which is essential for catalytic activity (18).

In the present study, we examined the association between oxidative stress and energy metabolism in TGF- $\beta 1$-induced EMT. We examined the expression of $\mathrm{Pkm} 2$ to reflect the changes in energy metabolism. Furthermore, we demonstrated the functionality of a pro-resolving lipid mediator, aspirin-triggered resolvin D1 (AT-RvD1), which is known to affect the resolution of inflammation $(19,20)$ by inhibiting TGF- $\beta 1$-induced EMT. This occurs through the inhibition of the mTOR pathway by reducing the expression of $\mathrm{Pkm} 2$.

\section{Materials and methods}

Reagents. Recombinant human TGF- $\beta 1$ was purchased from Peprotech, Inc. (Rocky Hill, NJ, USA). AT-RvD1 was purchased from Cayman Chemical Co. (Ann Arbor, MI, USA). Antibodies against Nrf2 (sc-722), $\beta$-actin (sc-7210 and Pkm2 (sc-135048) were obtained from Santa Cruz Biotechnology, Inc. (Santa Cruz, CA, USA). The mTOR activator MHY1485 was purchased from Millipore Corp. (Bedford, MA, USA). Fetal bovine serum (FBS) and Dulbecco's modified Eagle's medium (DMEM) were obtained from Gibco (Carlsbad, CA, USA).

Cell culture. The human lung adenocarcinoma epithelial cell line A549 was obtained from the Experimental Center of the Clinical Medicine College of Yangzhou University (Yangzhou, China). These cells were cultured in DMEM supplemented with $10 \%$ FBS, streptomycin and penicillin (Beyotime Institute of Biotechnology, Shanghai, China). The cells were grown at $37^{\circ} \mathrm{C}$ in a humidified $5 \% \mathrm{CO}_{2}$ atmosphere. The A549 cells were treated with different concentrations of TGF- $\beta 1$ (1,10 and $20 \mathrm{ng} / \mathrm{ml}$ ) for $48 \mathrm{~h}$. In order to examine the effects of AT-RvD1, the cells were pre-treated with AT-RvD1 for $30 \mathrm{~min}$ and then treated with TGF- $\beta 1$. In experiments using the mTOR activator, the cells were pre-treated with MHY1485 $(10 \mu \mathrm{mol} / \mathrm{ml})$ for $2 \mathrm{~h}$ and then treated with AT-RvD1 or TGF- $\beta 1$ as described above.

Measurement of cell proliferation. The cells were seeded in $100 \mu \mathrm{l}$ serum-free media with TGF- $\beta 1$ or AT-RvD1 for $48 \mathrm{~h}$ in 96-well plates. At $48 \mathrm{~h}$, cell proliferation was measured with an MTT cell proliferation assay kit (Beyotime Institute of Biotechnology) at an absorbance of $490 \mathrm{~nm}$ using a plate reader (3599; Corning, Corning, NY, USA). All measurements were performed in triplicate and the experiments were repeated three times.
Migration assay using scratch-wound healing. The cell cultures were prepared with different concentrations of TGF- $\beta 1$ or AT-RvD1 in 6-well plates to $80-90 \%$ confluence prior to performing the scratch-wound healing assay. The cells were washed with phosphate buffered saline (PBS) three times and cultured in serum-free medium. After incubating for $48 \mathrm{~h}$ at $37^{\circ} \mathrm{C}$, images of the cells that had migrated onto the scratches were captured and counted under a microscope (3516; Corning). All images were randomly acquired with a $100 \mathrm{X}$ objective at consistent intensity settings. We analyzed five fields of view for each experimental condition. The experiment was repeated three times.

Transwell invasion assay. Cell invasion was determined using 24-well plates with Transwell chamber inserts (Chemicon International, Inc., Atlanta, GA, USA), which had membranes coated with Matrigel. In the upper chambers, $3 \times 10^{4}$ A549 cells were seeded in FBS-free culture media, and media with $10 \%$ serum were added to the lower chambers. After a 24-h incubation, the cells on the upper surface of the membrane were scraped off, and the cells that had migrated onto the lower surface of the membrane were immersed in $500 \mu 1$ media with $0.5 \mathrm{mg} / \mathrm{ml}$ MTT for enumeration.

Measurement of ROS. Cellular ROS levels were determined using a ROS Assay kit (Beyotime Institute of Biotechnology). The cells in 96-well plates were treated with TGF- $\beta 1$ or AT-RvD1 for $48 \mathrm{~h}$. The cells were then incubated with $200 \mu 1$ DMEM and a 2',7'-dichlorofluorescein diacetate (DCFH-DA) fluorescent probe for $30 \mathrm{~min}$ at $37^{\circ} \mathrm{C}$. The fluorescence intensity was measured with a microplate reader at $488 \mathrm{~nm}$ excitation and $525 \mathrm{~nm}$ emission wavelengths. Moreover, a fluorescence microscope (Axio Observer A1, Zeiss, Oberkochen, Germany) was used to capture images.

Total RNA extraction and reverse transcription-quantitative polymerase chain reaction $(R T-q P C R)$. Total RNA was extracted from cells using TRIzol reagent (Invitrogen, Carlsbad, USA) according to the manufacturer's instructions. The reverse transcription of $1 \mu \mathrm{g}$ RNA was performed using qRT SuperMix (Vazyme, Nanjing, China). RT-qPCR was performed using the ABI StepOne Plus system (Applied Biosystems Life Technologies, Foster City, CA, USA) with the AceQ SYBR-Green Master Mix (Vazyme). The primers were synthesized by Bioneer Corp. (Daejeon, Korea), and the following primer sequences for the human genes were used: E-cadherin, 5'-AAAGGCCCATTTCCTAAAAACCT-3' and 5'-TGCGTTCTCTATCCAGAGGCT-3'; vimentin, 5'-TGCCG TTGAAGCTGCTAACTA-3' and 5'-CCAGAGGGAGTGAA TCCAGATTA-3'; Nrf2, 5'-TTCCCGGTCACATCGAGAG-3' and 5'-TCCTGTTGCATACCGTCTAAATC-3'; Pkm2, 5'-ATG TCGAAGCCCCATAGTGAA-3' and 5'-TGGGTGGTGAAT CAATGTCCA-3'; and $\beta$-actin, 5'-AAGACCTGTACGCCAA CACAGT-3' and 5'-AGAAGCATTTGCGGTGGACGAT-3'. The amplified expression of gene transcriptions were normalized to $\beta$-actin expression. The following PCR conditions were used: holding stage, $95^{\circ} \mathrm{C}$ for $5 \mathrm{~min}$; cycling stage, $95^{\circ} \mathrm{C}$ for $10 \mathrm{sec}$ and $60^{\circ} \mathrm{C}$ for $30 \mathrm{sec} 40$ times; melt curve stage, $95^{\circ} \mathrm{C}$ for $15 \mathrm{sec}, 60^{\circ} \mathrm{C}$ for $60 \mathrm{sec}$ and $95^{\circ} \mathrm{C}$ for $15 \mathrm{sec}$. Cycle threshold $(\Delta \Delta \mathrm{Ct})$ values were calculated for each 

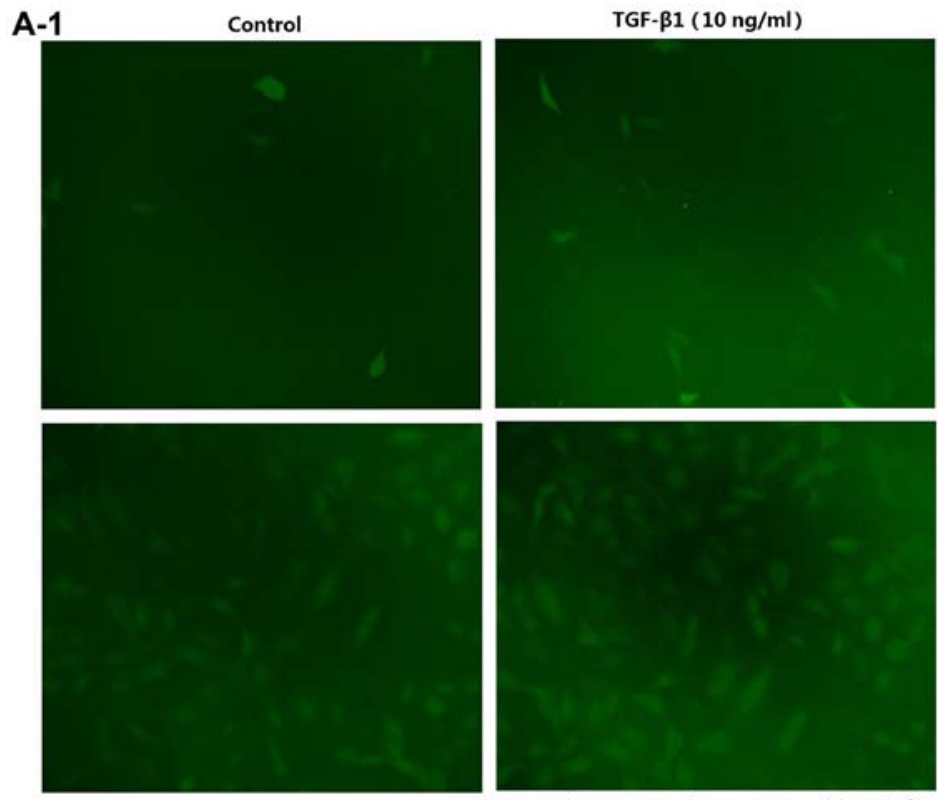

TGF- $\beta 1(20 \mathrm{ng} / \mathrm{ml})$

TGF- $\beta 1(20 \mathrm{ng} / \mathrm{ml})+$ AT-RvD1 $(20 \mathrm{ng} / \mathrm{ml})$

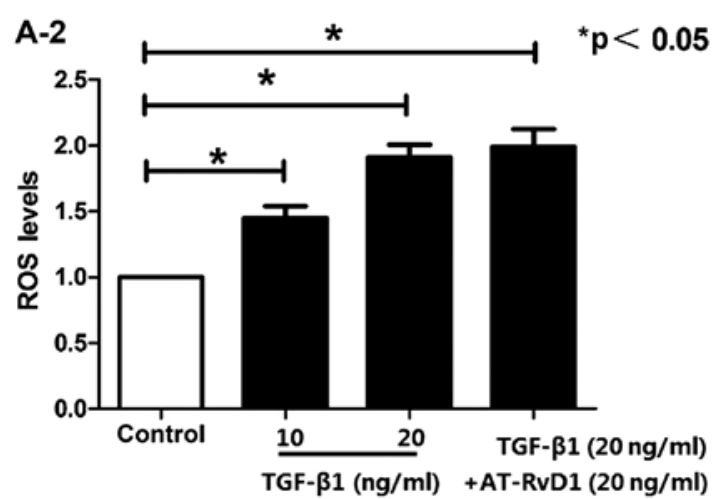

B

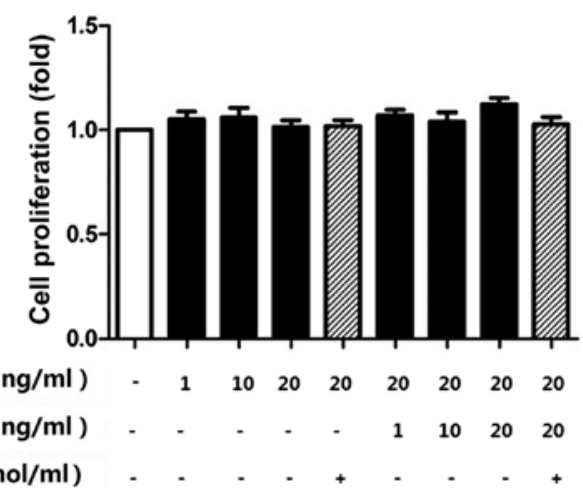

Figure 1. AT-RvD1 inhibition of TGF- $\beta 1$-induced EMT is associated with ROS and Pkm2. (A1 and 2) Determination of ROS levels. Cells were pre-treated with AT-RvD1 for $30 \mathrm{~min}$. The A549 cells in 96-well plates were then treated with different concentrations of TGF- $\beta 1$ (1, $10 \mathrm{and} 20 \mathrm{ng} / \mathrm{ml})$ for $48 \mathrm{~h}$. We used an ROS assay kit, a microplate reader and a fluorescence microscope as described in the Materials and methods. Images in A1, x100 magnification. (B) Effects of AT-RvD1, TGF- $\beta 1$ and mTOR activator on the proliferation of A549 cells. The results showed that AT-RvD1 and TGF- $\beta 1$ had no effect on cell proliferation. AT-RvD1, aspirin-triggered resolvin D1; TGF- $\beta 1$, transforming growth factor- $\beta 1$; EMT, epithelial-to-mesenchymal transition; ROS, reactive oxygen species; Pkm2, pyruvate kinase M2.

experimental group, which indicated the number of available template cDNA in each reaction. The gene expression levels were compared using the values of $2^{-\Delta \Delta \mathrm{Ct}}$.

Total protein extraction and western blot analysis. The total protein extracted from cells was analyzed using a radioimmunoprecipitation assay (RIPA) and phenylmethylsulfonyl fluoride (PMSF) (Beyotime Institute of Biotechnology). Protein concentrations were measured using the bicinchoninic acid (BCA) assay. The protein samples were separated by electrophoresis on $6-10 \%$ sodium dodecyl sulfate (SDS)-polyacrylamide gels and transferred to polyvinylidene fluoride (PVDF) membranes (Invitrogen). The membranes were blocked with $5 \%$ skim milk for $2 \mathrm{~h}$ and then incubated with the appropriate antibodies against $\mathrm{Nrf} 2, \mathrm{Pkm} 2$ and $\beta$-actin (Santa Cruz Biotechnology, Inc.) in $4 \%$ bovine serum albumin (BSA) at $4^{\circ} \mathrm{C}$ overnight. The membranes were further incubated for $2 \mathrm{~h}$ with a peroxidase-conjugated secondary antibody (Beyotime Institute of Biotechnology) at room temperature, and the results were detected using a ChemiDoc imaging system (Bio-Rad Laboratories, Inc., Hercules, CA, USA) and analyzed using ImageJ software.

Statistical analysis. All data are expressed as the means \pm SEM and were analyzed by one-way analysis of variance (ANOVA) followed by the Newman-Keuls multiple comparison test (GraphPad Prism software; GraphPad Software, Inc., La Jolla, CA, USA). Data were recorded from at least three independent experiments. A p-value $<0.05$ was considered to indicate a statistically significant difference.

\section{Results}

TGF- $\beta 1$-induced EMT increases ROS levels and the expression of Pkm2 in A549 cells. TGF- $\beta 1$-induced EMT in the A549 cells enhanced the mRNA expression of the mesenchymal marker vimentin, and reduced the expression of the epithelial marker E-cadherin (Fig. 1D). These findings were consistent with those of previous studies $(13,21)$. The ROS levels were significantly increased in the A549 cells following 

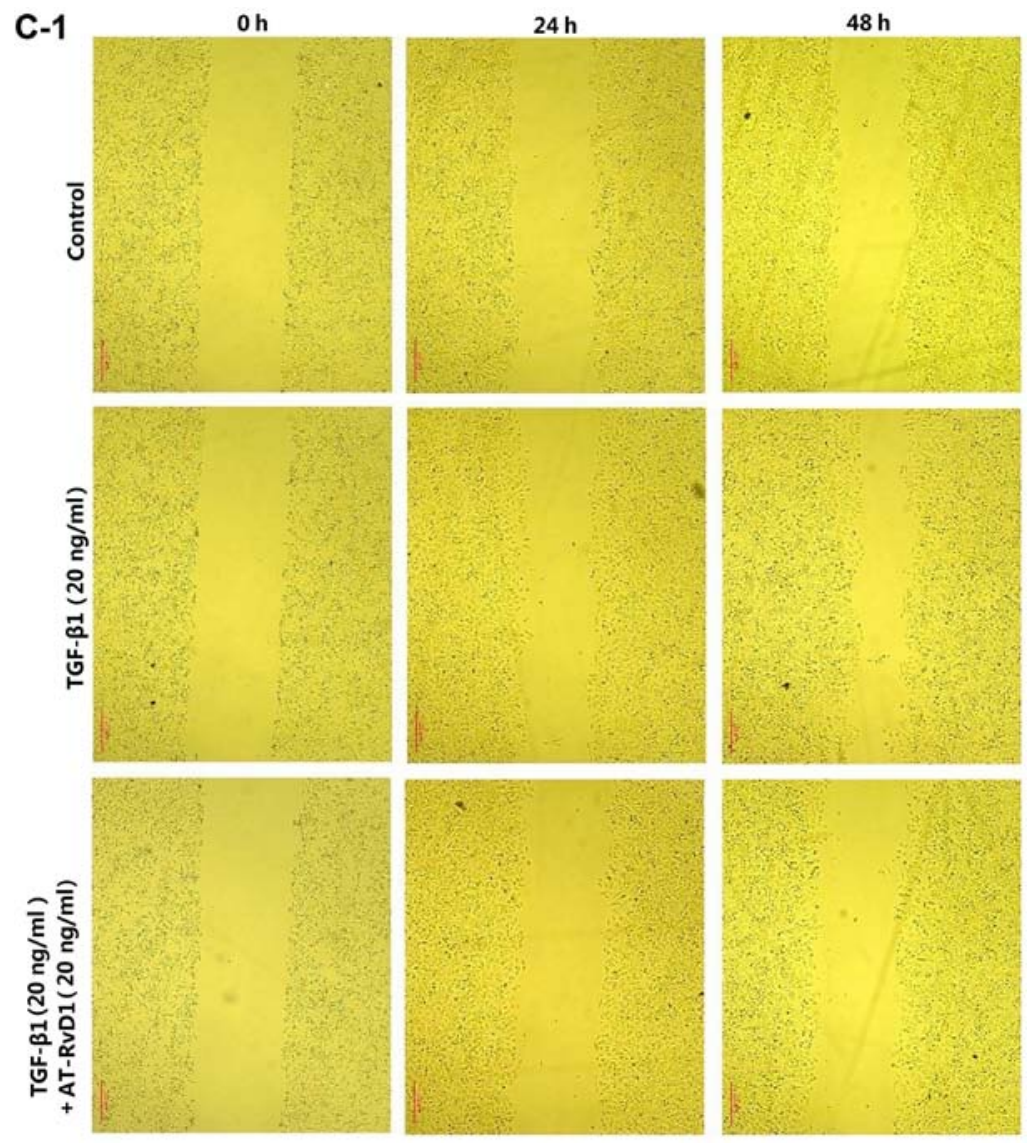

C-2

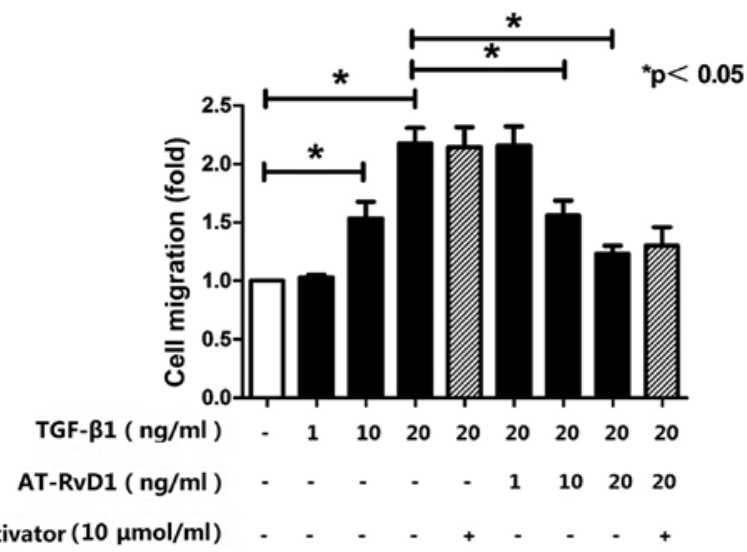

C-3

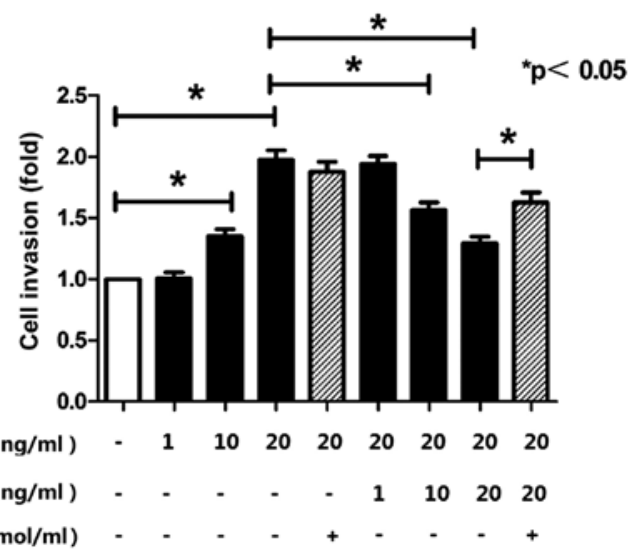

Figure 1. Continued. AT-RvD1 inhibition of TGF- $\beta 1$-induced EMT is associated with ROS and Pkm2. (C1-3) Effects of AT-RvD1 on TGF- $\beta 1$-induced cell migration and invasion. The results showed that AT-RvD1 in combinaton with TGF- $\beta 1$ inhibited TGF- $\beta 1$-induced cell migration and invasion. AT-RvD1, aspirin-triggered resolvin D1; TGF- $\beta 1$, transforming growth factor- $\beta 1$; EMT, epithelial-to-mesenchymal transition; ROS, reactive oxygen species; Pkm2, pyruvate kinase M2.

incubation with TGF- $\beta 1$ in a concentration-dependent manner, with $20 \mathrm{ng} / \mathrm{ml}$ TGF- $\beta 1$ significantly elevating the levels of ROS (Fig. 1A). The migration and invasion of A549 cells were enhanced by TGF- $\beta 1$ (Fig. 1C). It has been previously demonstrated that Nrf2 plays a critical role in the removal of cellular ROS, and TGF- $\beta 1$ is capable of reducing Nrf2 expression in renal epithelial cells (16). The effect of TGF- $\beta 1$ on Nrf2 expression in A549 cells was also confirmed by RT-qPCR and western blot analyses (Fig. 1D and E). In addition, the expression of Pkm2, which plays a crucial role in tumor metabolism, was increased by exposure to TGF- $\beta 1$ (Fig. 1D and E). These results suggest that TGF- $\beta 1$-induced EMT increased ROS levels and the expression of $\mathrm{Pkm} 2$ in A549 cells.
AT-RvDl inhibits TGF- $\beta 1$-induced EMT through inhibition of the mTOR pathway by reducing the expression of Pkm2. To elucidate the mechanism responsible for the effects of AT-RvD1 on the TGF- $\beta 1$-induced EMT process, the cells were treated with different concentrations of AT-RvD1 30 min prior to the addition of TGF- $\beta 1$. AT-RvD1 treatment blocked the expression of the mesenchymal marker vimentin and restored the expression of E-cadherin. The effects of AT-RvD1 on the expression of E-cadherin and vimentin in TGF- $\beta 1$-treated A549 cells were confirmed using RT-qPCR (Fig. 2A). However, AT-RvD1 did not reduce the ROS levels in the TGF- $\beta 1$-treated A549 cells (Fig. 1A). The reduced expression of Nrf2 in the TGF- $\beta 1$-treated A549 cells was further lowered 

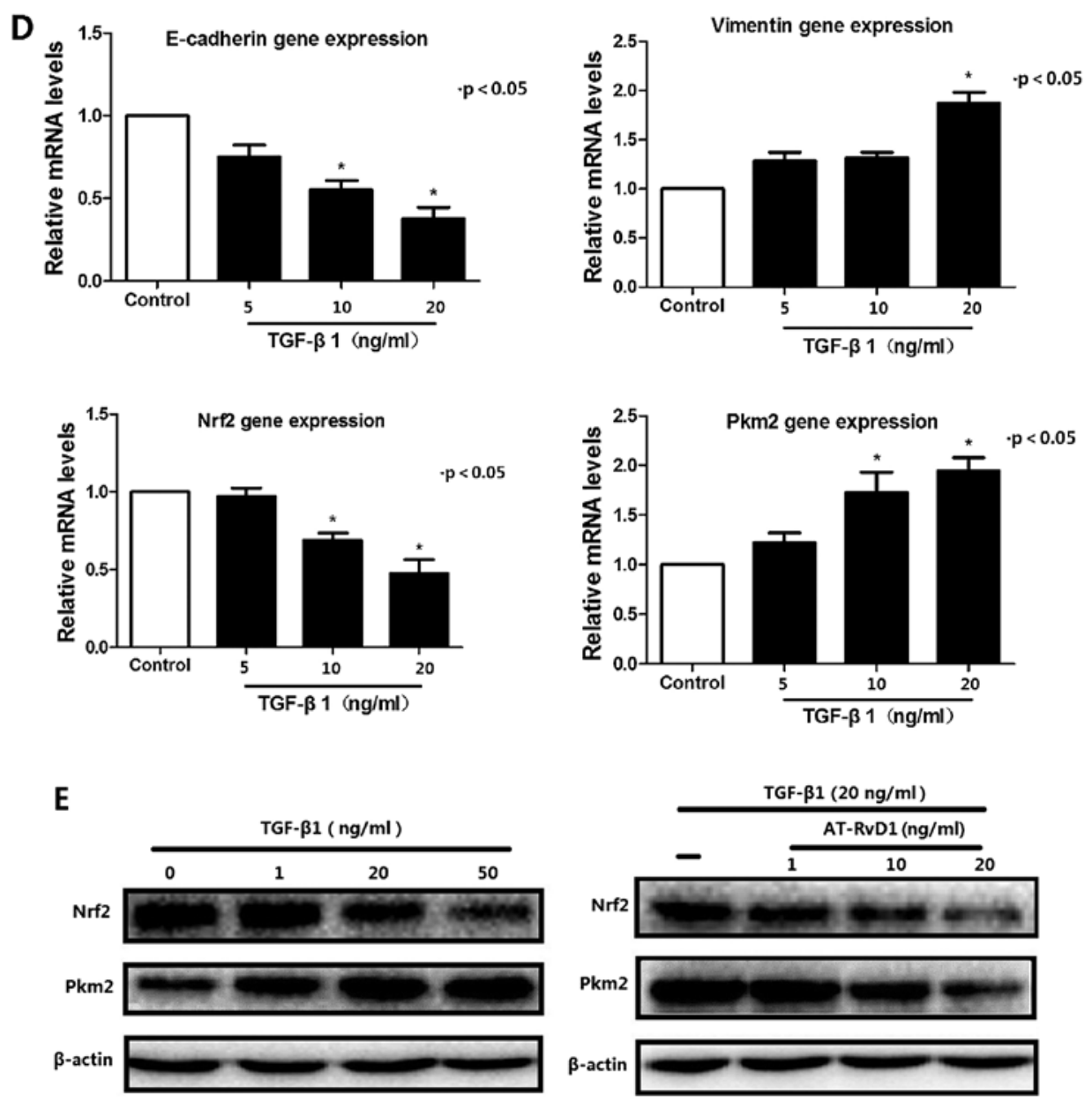

Figure 1. Continued. AT-RvD1 inhibition of TGF- $\beta 1$-induced EMT is associated with ROS and Pkm2. (D) RT-qPCR of EMT markers in A549 cells treated with TGF- $\beta 1(0-20 \mathrm{ng} / \mathrm{ml})$. (E) Western blot analysis of the expression of Nrf2 and Pkm2. $\beta$-actin was used as a control. The values were normalized to each control and the means \pm SE from three independent experiments are presented. ${ }^{*} \mathrm{p}<0.05$ compared with control groups. AT-RvD1, aspirin-triggered resolvin D1; TGF- $\beta 1$, transforming growth factor- $\beta 1$; EMT, epithelial-to-mesenchymal transition; ROS, reactive oxygen species; Pkm2, pyruvate kinase M2; Nrf2, nuclear factor (erythroid-derived 2)-like 2.

by AT-RvD1 (Fig. 1E). However, AT-RvD1 clearly reduced the concentration-dependent TGF- $\beta 1$-induced migration and invasion (Fig. 1C) but did not affect the proliferative properties of the TGF- $\beta 1$-treated A549 cells (Fig. 1B). Our experiments have conclusively shown that the mTOR activator had no effect on the TGF- $\beta 1$-induced EMT. However, the activation of mTOR signaling restored the expression of $\mathrm{Pkm} 2$, which was suppressed by AT-RvD1, and partly downregulated the expression of E-cadherin (Fig. 2). The inhibitory effect of AT-RvD1 on the TGF- $\beta 1$-induced EMT was weakened by mTOR activator.

These results suggest that AT-RvD1 inhibited TGF- $\beta 1$-induced EMT through the inhibition of the mTOR pathway by reducing the expression of $\mathrm{Pkm} 2$.

\section{Discussion}

Cancer, similar to chronic respiratory diseases and diabetes, is a noncommunicable disease. It is characterized by a long duration and slow development (3). The high mortality rates associated with cancer are caused by tumor cell metastases. In fact, metastases are the primary causes of $90 \%$ of cancer deaths (22). The EMT is involved in tumor cell metastasis. The
EMT process is directly associated with the features of the primary tumor microenvironment (23). A molecular feature of the EMT is the downregulation of epithelial markers and the upregulation of a number of mesenchymal markers (24).

The tumor stroma is composed of an extracellular matrix, vasculature, lymphatic systems and other cells. Changes to these cell phenotypes are associated with tumorigenesis and metastasis. The resultant cells are tumor-associated fibroblasts and macrophages $(21,25)$. Several inflammatory mediators are released during these changes to the tumor microenvironment, leading to the progression and metastasis of the tumor (21). Specialized pro-resolving lipid mediators, such as lipoxin and resolvin, due to their involvement in the inhibitory process of EMT $(21,27)$, have the ability to prevent excessive neutrophil trafficking to inflammation sites and thereby promote the recovery of damaged tissue $(20,26,27)$. However, the role of AT-RvD1 remains unclear. Thus, we examined the effects of the available AT-RvD1 on the TGF- $\beta 1$-induced EMT of lung cancer cells.

AT-RvD1 suppressed the TGF- $\beta 1$-regulated expression of vimentin and restored the expression of E-cadherin in A549 lung cancer cells. However, the effects of AT-RvD1 are not associated with cell proliferation, which is one of the 
A
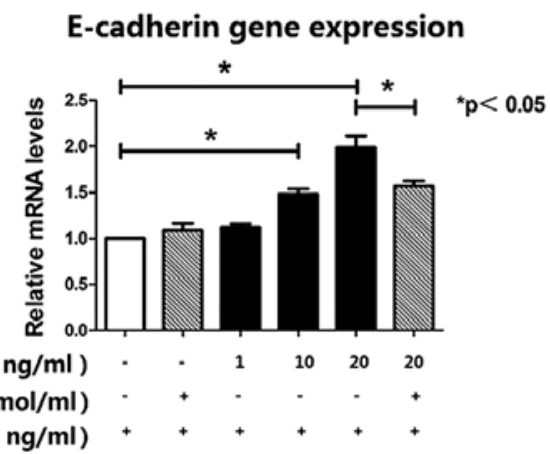

Nrf2 gene expression

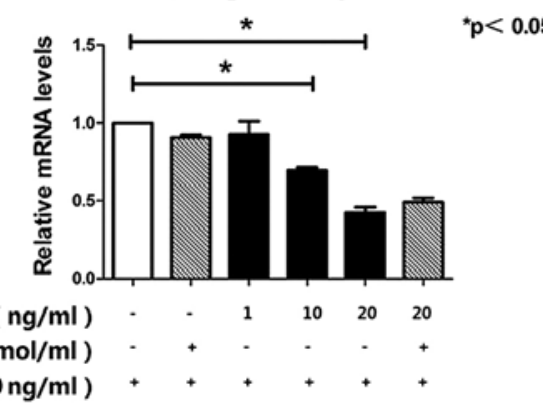

Vimentin gene expression

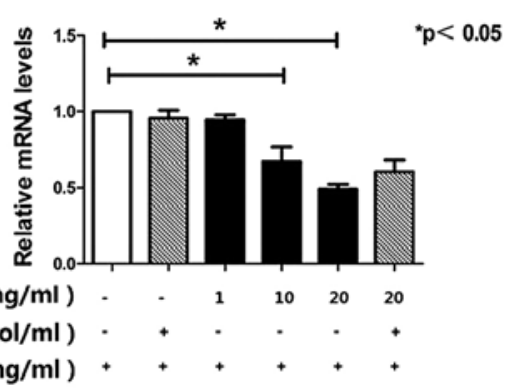

Pkm2 gene expression

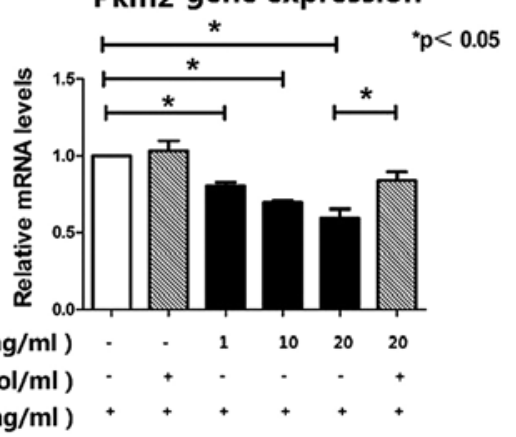

B

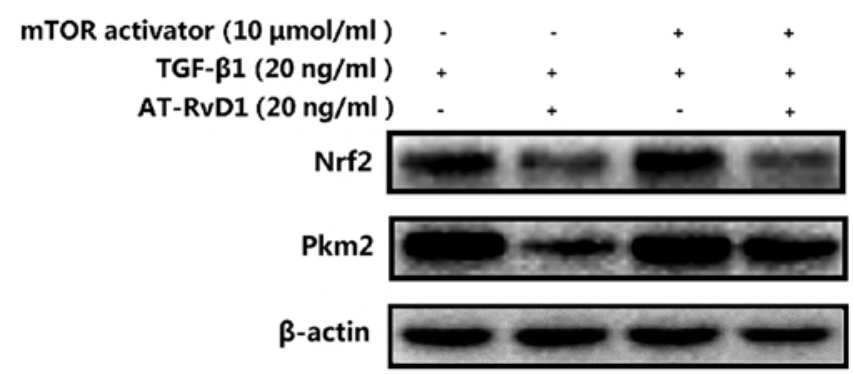

Figure 2. AT-RvD1 inhibits TGF- $\beta 1$-induced EMT through inhibition of the mTOR pathway by reducing the expression of PKM2. (A) Cells were pre-treated with mTOR activator $(10 \mu \mathrm{mol} / \mathrm{ml})$ for $2 \mathrm{~h}$. The A549 cells were then treated with AT-RvD1 and TGF- $\beta 1$ as shown above. The effects of mTOR activator on the EMT markers are shown. The effects of AT-RvD1 on the gene expression of Nrf2 and Pkm 2 in the TGF- $\beta 1$-induced EMT process were altered. (B) Cells were treated with an mTOR activator for $2 \mathrm{~h}$. The cells were then cultured in TGF- $\beta 1(20 \mathrm{ng} / \mathrm{ml})$ and AT-RvD1 $(20 \mathrm{ng} / \mathrm{ml})$. After incubation, the protein expression of Nrf 2 and Pkm2 was determined by western blot analysis. AT-RvD1, aspirin-triggered resolvin D1; TGF- $\beta 1$, transforming growth factor- $\beta 1$; EMT, epithelial-to-mesenchymal transition; ROS, reactive oxygen species; Pkm2, pyruvate kinase M2; Nrf2, nuclear factor (erythroid-derived 2)-like 2.

characteristics of the EMT. Moreover, AT-RvD1 also inhibited the TGF- $\beta 1$-induced migration and invasion of A549 cells. A previous study (21) has shown that resolvin (Rv)D1 and RvD2 both suppressed TGF- $\beta 1$-induced EMT, and RvD1 inhibited the TGF- $\beta 1$-induced EMT through the lipoxin A4 receptor/formyl peptide receptor 2 (ALX/FPR2) and G protein-coupled receptor 32 (GPR32) receptors by reducing the expression of ZEB1. Lipoxin A4 (LXA4), another mediator of resolution, has also been reported to inhibit the connective tissue growth factor (CTGF)-induced EMT process (27). Our results have shown that AT-RvD1 may also be useful in suppressing the EMT of lung cancer cells. Our future studies will explore the association between the EMT process and oxidative stress.

The effects of TGF- $\beta 1$ and AT-RvD1 correlate with the high levels of ROS in A549 lung cancer cells. ROS have been reported to promote the TGF- $\beta 1$-induced EMT genetic changes (16). However, high concentrations of ROS may also damage cellular components and compromise cell viability (18). Acute increases in the intracellular concentrations of ROS have been found to inhibit the glycolytic enzyme $\mathrm{Pkm} 2$ in lung cancer cells (18). Pkm2 enables proliferating cells to divert glucose into anabolic pathways to meet the increased biosynthetic demands of proliferation (18). Thus, TGF- $\beta 1$-induced ROS may be associated with cell proliferation. The inhibitory effect of TGF- $\beta 1$ on cell proliferation is usually on normal cells and some types of cancer cell, and is associated with the concentration of TGF- $\beta 1$ and some cancer-specific factors, so in our study TGF- $\beta 1$ induced the migration of the cells but had no effect on cell proliferation.

We also examined the expression of $\mathrm{Nrf2}$, which is one of the key proteins involved in antioxidant signaling pathways. Both TGF- $\beta 1$ and AT-RvD1 suppressed the expression of Nrf2. The antioxidant signaling pathway Keap1-Nrf2 is known to have protective effects in both normal and cancer cells (28). With the increase in ROS levels, the activation of the antioxidant response element (ARE) reporter has been found to lead 
to elevated levels of GSH, which enable NADPH to sustain cell proliferation $(20,29,30)$. The low expression of Nrf2 weakens the cell protection and leads to increased ROS levels.

We also examined the elevated expression of $\mathrm{Pkm} 2$ during the TGF- $\beta 1$-induced EMT. Treatment with AT-RvD1 reduced the expression of Pkm2 in A549 cells. Glucose is also a major carbon source for biosynthetic processes, and $\mathrm{Pkm} 2$ is known to partially determine how glucose is metabolized in cancer cells (31). Pkm2 is commonly upregulated in many types of human cancer and has been shown to play a crucial role in reprogramming cellular metabolism, gene transcription and cell cycle progression $(26,32)$. A study (17) demonstrated that the EMT stimulates the nuclear translocation of $\mathrm{Pkm} 2$ and decreases E-cadherin transcription. $\mathrm{Pkm} 2$ interacts with transcription factor TGF- $\beta$-induced factor homeobox 2 (TGIF2), which induces the deacetylation of histone $\mathrm{H} 3$, resulting in the repressed expression of E-cadherin. The discovery that AT-RvD1 reduces the expression of $\mathrm{Pkm} 2$ is notable, and the the inhibition of TGF- $\beta 1$-induced EMT by AT-RvD1 is possibly due to the reduced expression of $\mathrm{Pkm} 2$. Further studies are necessary in order to explore the associated mechanisms.

The mTOR pathway is known to regulate the expression of various glycolytic enzymes including Pkm2 $(33,34)$. The inhibition of mTOR also reduces Pkm2 expression (35). Thus, we examined the effects of mTOR activator on changes made by AT-RvD1 to the TGF- $\beta 1$-induced EMT and found that there was an association. The mTOR activator did restore the expression of Pkm2, which had been suppressed by AT-RvD1, and subsequently downregulated the expression of E-cadherin. The inhibitory effect of AT-RvD1 on the TGF- $\beta 1$-induced EMT was weakened. However, increased oxidative stress is known to inhibit the phosphoinositide 3-kinase (P13K)/AKT/mTOR pathways through the disruption of mTORC1 formation (36). This may potentially explain the means through which increased oxidative stress affects cellular energy metabolism. AT-RvD1 maintained a high level of oxidative stress and reduced the expression of $\mathrm{Pkm} 2$ whereas it had no effect on cell proliferation, thus, the mechanism may be associated with a particular receptor (37). These results suggest that AT-RvD1 inhibited the TGF- $\beta 1$-induced EMT through the inhibition of the mTOR pathway by reducing the expression of Pkm2.

There are a few limitations to our experiments. We illustrated the association between energy metabolism and oxidative stress; however, the dynamics are complex and require further investigation. In addition, the effect of AT-RvD1 has not yet been confirmed in in vivo animal cancer models. In future studies, we will focus our efforts on exploring other mechanisms responsible for the low expression of $\mathrm{Pkm} 2$ mediated by AT-RvD1 and also perform animal experiments.

Our results suggest the possibility that AT-RvD1 suppresses the TGF- $\beta 1$-induced EMT by affecting cellular energy metabolism and oxidative stress.

\section{References}

1. Parkin DM, Bray F, Ferlay J and Pisani P: Global cancer statistics, 2002. CA Cancer J Clin 55: 74-108, 2005.

2. Williams BA, Sugimura H, Endo C, Nichols FC, Cassivi SD, Allen MS, Pairolero PC, Deschamps C and Yang P: Predicting postrecurrence survival among completely resected non-small-cell lung cancer patients. Ann Thorac Surg 81: $1021-1027,2006$
3. Okada F: Inflammation-related carcinogenesis: current findings in epidemiological trends, causes and mechanisms. Yonago Acta Med 57: 65-72, 2014.

4. Loomis D, Grosse Y, Lauby-Secretan B, El Ghissassi F, Bouvard V, Benbrahim-Tallaa L, Guha N, Baan R, Mattock H and Straif K; International Agency for Research on Cancer Monograph Working Group IARC: The carcinogenicity of outdoor air pollution. Lancet Oncol 14: 1262-1263, 2013.

5. Tazawa H, Okada F, Kobayashi T, Tada M, Mori Y, Une Y, Sendo F, Kobayashi M and Hosokawa M: Infiltration of neutrophils is required for acquisition of metastatic phenotype of benign murine fibrosarcoma cells: implication of inflammation-associated carcinogenesis and tumor progression. Am J Pathol 163: 2221-2232, 2003

6. Okada F, Kobayashi M, Tanaka H, Kobayashi T, Tazawa H, Iuchi Y, Onuma K, Hosokawa M, Dinauer MC and Hunt NH: The role of nicotinamide adenine dinucleotide phosphate oxidase-derived reactive oxygen species in the acquisition of metastatic ability of tumor cells. Am J Pathol 169: 294-302, 2006.

7. Okada F, Tazawa H, Kobayashi T, Kobayashi M and Hosokawa M: Involvement of reactive nitrogen oxides for acquisition of metastatic properties of benign tumors in a model of inflammation-based tumor progression. Nitric Oxide 14: 122-129, 2006.

8. Kalluri R and Weinberg RA: The basics of epithelial-mesenchymal transition. J Clin Invest 119: 1420-1428, 2009.

9. Gemmill RM, Roche J, Potiron VA, Nasarre P, Mitas M, Coldren CD, Helfrich BA, Garrett-Mayer E, Bunn PA and Drabkin HA: ZEB1-responsive genes in non-small cell lung cancer. Cancer Lett 300: 66-78, 2011.

10. Prudkin L, Liu DD, Ozburn NC, Sun M, Behrens C, Tang X, Brown KC, Bekele BN, Moran C and Wistuba II: Epithelial-to-mesenchymal transition in the development and progression of adenocarcinoma and squamous cell carcinoma of the lung. Mod Pathol 22: 668-678, 2009.

11. Pohlers D, Brenmoehl J, Löffler I, Müller CK, Leipner C, Schultze-Mosgau S, Stallmach A, Kinne RW and Wolf G: TGF- $\beta$ and fibrosis in different organs - molecular pathway imprints. Biochim Biophys Acta 1792: 746-756, 2009.

12. Kawata M, Koinuma D, Ogami T, Umezawa K, Iwata C, Watabe T and Miyazono K: TGF- $\beta$-induced epithelial-mesenchymal transition of A549 lung adenocarcinoma cells is enhanced by pro-inflammatory cytokines derived from RAW 264.7 macrophage cells. J Biochem 151: 205-216, 2012.

13. Park MK, You HJ, Lee HJ, Kang JH, Oh SH, Kim SY and Lee $\mathrm{CH}$ : Transglutaminase-2 induces $\mathrm{N}$-cadherin expression in TGF- $\beta 1$-induced epithelial mesenchymal transition via c-Jun-N-terminal kinase activation by protein phosphatase $2 \mathrm{~A}$ down-regulation. Eur J Cancer 49: 1692-1705, 2013.

14. Kashihara N, Haruna Y, Kondeti VK and Kanwar YS: Oxidative stress in diabetic nephropathy. Curr Med Chem 17: 4256-4269, 2010.

15. Kobayashi $\mathbf{M}$ and Yamamoto M: Molecular mechanisms activating the Nrf2-Keap1 pathway of antioxidant gene regulation. Antioxid Redox Signal 7: 385-394, 2005.

16. Ryoo IG, Ha $\mathrm{H}$ and Kwak MK: Inhibitory role of the KEAP1-NRF2 pathway in TGF $\beta 1$-stimulated renal epithelial transition to fibroblastic cells: a modulatory effect on SMAD signaling. PLoS One 9: e93265, 2014.

17. Hamabe A, Konno M, Tanuma N, Shima H, Tsunekuni K, Kawamoto K, Nishida N, Koseki J, Mimori K, Gotoh N, et al: Role of pyruvate kinase M2 in transcriptional regulation leading to epithelial-mesenchymal transition. Proc Natl Acad Sci USA 111: 15526-15531, 2014.

18. Anastasiou D, Poulogiannis G, Asara JM, Boxer MB, Jiang JK, Shen M, Bellinger G, Sasaki AT, Locasale JW, Auld DS, et al: Inhibition of pyruvate kinase M2 by reactive oxygen species contributes to cellular antioxidant responses. Science 334: 1278-1283, 2011.

19. Fadok VA, Bratton DL, Konowal A, Freed PW, Westcott JY and Henson PM: Macrophages that have ingested apoptotic cells in vitro inhibit proinflammatory cytokine production through autocrine/paracrine mechanisms involving TGF-beta, PGE2, and PAF. J Clin Invest 101: 890-898, 1998.

20. Serhan CN, Hong S, Gronert K, Colgan SP, Devchand PR, Mirick G and Moussignac RL: Resolvins: a family of bioactive products of omega-3 fatty acid transformation circuits initiated by aspirin treatment that counter proinflammation signals. J Exp Med 196: 1025-1037, 2002. 
21. Lee HJ, Park MK, Lee EJ and Lee CH: Resolvin D1 inhibits TGF- $\beta 1$-induced epithelial mesenchymal transition of A549 lung cancer cells via lipoxin A4 receptor/formyl peptide receptor 2 and GPR32. Int J Biochem Cell Biol 45: 2801-2807, 2013.

22. Steeg PS: Tumor metastasis: mechanistic insights and clinical challenges. Nat Med 12: 895-904, 2006.

23. Neil JR, Johnson KM, Nemenoff RA and Schiemann WP: Cox-2 inactivates Smad signaling and enhances EMT stimulated by TGF- $\beta$ through a PGE2-dependent mechanisms. Carcinogenesis 29: 2227-2235, 2008.

24. Talbot LJ, Bhattacharya SD and Kuo PC: Epithelial-mesenchymal transition, the tumor microenvironment, and metastatic behavior of epithelial malignancies. Int J Biochem Mol Biol 3: 117-136, 2012.

25. Yang W and Lu Z: Pyruvate kinase M2 at a glance. J Cell Sci 128: $1655-1560,2015$.

26. Spite M, Norling LV, Summers L, Yang R, Cooper D, Petasis NA, Flower RJ, Perretti M and Serhan CN: Resolvin D2 is a potent regulator of leukocytes and controls microbial sepsis. Nature 461: 1287-1291, 2009

27. Wu SH, Zhang YM, Tao HX and Dong L: Lipoxin A(4) inhibits transition of epithelial to mesenchymal cells in proximal tubules. Am J Nephrol 32: 122-136, 2010.

28. Homma S, Ishii Y, Morishima Y, Yamadori T, Matsuno Y, Haraguchi N, Kikuchi N, Satoh H, Sakamoto T, Hizawa N, et al: Nrf2 enhances cell proliferation and resistance to anticancer drugs in human lung cancer. Clin Cancer Res 15: 3423-3432, 2009.

29. Brown SL, Sekhar KR, Rachakonda G, Sasi S and Freeman ML: Activating transcription factor 3 is a novel repressor of the nuclear factor erythroid-derived 2-related factor 2 (Nrf2)-regulated stress pathway. Cancer Res 68: 364-368, 2008.

30. Dhakshinamoorthy S, Jain AK, Bloom DA and Jaiswal AK: Bach1 competes with Nrf2 leading to negative regulation of the antioxidant response element (ARE)-mediated NAD(P) $\mathrm{H}$ :quinone oxidoreductase 1 gene expression and induction in response to antioxidants. J Biol Chem 280: 16891-16900, 2005.
31. Christofk HR, Vander Heiden MG, Harris MH, Ramanathan A, Gerszten RE, Wei R, Fleming MD, Schreiber SL and Cantley LC: The M2 splice isoform of pyruvate kinase is important for cancer metabolism and tumour growth. Nature 452: 230-233, 2008.

32. Yang W, Xia Y, Cao Y, Zheng Y, Bu W, Zhang L, You MJ, Koh MY, Cote G, Aldape K, et al: EGFR-induced and PKCe monoubiquitylation-dependent $\mathrm{NF}-\kappa \mathrm{B}$ activation upregulates PKM2 expression and promotes tumorigenesis. Mol Cell 48: 771-784, 2012.

33. Majumder PK, Febbo PG, Bikoff R, Berger R, Xue Q, McMahon LM, Manola J, Brugarolas J, McDonnell TJ, Golub TR, et al: mTOR inhibition reverses Akt-dependent prostate intraepithelial neoplasia through regulation of apoptotic and HIF-1-dependent pathways. Nat Med 10: 594-601, 2004.

34. Sun Q, Chen X, Ma J, Peng H, Wang F, Zha X, Wang Y, Jing Y, Yang H, Chen R, et al: Mammalian target of rapamycin up-regulation of pyruvate kinase isoenzyme type M2 is critical for aerobic glycolysis and tumor growth. Proc Natl Acad Sci USA 108: 4129-4134, 2011.

35. Iqbal MA and Bamezai RNK: Resveratrol inhibits cancer cell metabolism by down regulating pyruvate kinase M2 via inhibition of mammalian target of rapamycin. PLoS One 7: e36764, 2012.

36. Hambright HG, Meng P, Kumar AP and Ghosh R: Inhibition of PI3K/AKT/mTOR axis disrupts oxidative stress-mediated survival of melanoma cells. Oncotarget 6: 7195-7208, 2015.

37. Al-Zaubai N, Johnstone CN, Leong MM, Li J, Rizzacasa M and Stewart AG: Resolvin D2 supports MCF-7 cell proliferation via activation of estrogen receptor. J Pharmacol Exp Ther 351: 172-180, 2014. 